\title{
Sharp Weak Type Inequality for Fractional Integral Operators Associated with $d$-Dimensional Walsh-Fourier Series
}

\author{
Adam Osȩkowski
}

\begin{abstract}
Suppose that $d \geq 1$ is an integer, $\alpha \in(0, d)$ is a fixed parameter and let $I_{\alpha}$ be the fractional integral operator associated with $d$-dimensional Walsh-Fourier series on $[0,1)^{d}$. The paper contains the proof of the sharp weak-type estimate
\end{abstract}

$$
\left\|I_{\alpha}(f)\right\|_{L^{d /(d-\alpha), \infty}\left([0,1)^{d}\right)} \leq \frac{2^{d}-1}{\left(2^{d-\alpha}-1\right)\left(2^{\alpha}-1\right)}\|f\|_{L^{1}\left([0,1)^{d}\right)} .
$$

The proof rests on Bellman-function-type method: the above estimate is deduced from the existence of a certain family of special functions.

Mathematics Subject Classification (2010). Primary 42B25, 42B30; Secondary 42B35.

Keywords. Fractional, best constant, weak-type inequality, Bellman function.

\section{Introduction}

Our motivation comes from the very natural question about sharp versions of estimates for $d$-dimensional Walsh system. As evidenced in numerous papers, such inequalities play an important role in many areas of mathematics, including approximation theory, Fourier analysis, harmonic analysis and probability theory. We refer the interested reader to the works $[2,5,20,21,23,24]$ and references therein.

Let us start with introducing the necessary background and notation. We will work with functions defined on the unit cube $[0,1)^{d}$ in $\mathbb{R}^{d}$, equipped with its dyadic sub-cubes, i.e., the sets of the form $\left[\frac{a_{1}}{2^{n}}, \frac{a_{1}+1}{2^{n}}\right) \times\left[\frac{a_{2}}{2^{n}}, \frac{a_{2}+1}{2^{n}}\right) \times$ $\ldots\left[\frac{a_{d}}{2^{n}}, \frac{a_{d}+1}{2^{n}}\right)$ for some nonnegative integer $n$ and some $a_{1}, a_{2}, \ldots, a_{d} \in$ $\left\{0,1, \ldots, 2^{n}-1\right\}$. Recall that the Rademacher system $\left\{r_{n}\right\}_{n \geq 0}$ of functions on $[0,1)$ is given by

$$
r_{n}(t)=\operatorname{sgn}\left(\sin \left(2^{n+1} \pi t\right)\right) .
$$


Then $\left\{w_{n}\right\}_{n \geq 0}$, the Walsh system on $[0,1)$, is defined as follows: $w_{0} \equiv 1$ and if $n$ is a positive integer with $n=2^{n_{1}}+2^{n_{2}}+\ldots+2^{n_{k}}$ and $n_{1}>n_{2}>\ldots>n_{k}$, then

$$
w_{n}(t)=r_{n_{1}}(t) r_{n_{2}}(t) \ldots r_{n_{k}}(t) .
$$

The $d$-dimensional counterpart of the Walsh system is the collection of all functions on $[0,1)^{d}$ which are of the form

$$
x=\left(x_{1}, x_{2}, \ldots, x_{d}\right) \mapsto w_{j_{1}}\left(x_{1}\right) w_{j_{2}}\left(x_{2}\right) \ldots w_{j_{d}}\left(x_{d}\right),
$$

where $j_{1}, j_{2}, \ldots, j_{d}$ are nonnegative integers.

Now, assume that $f$ is an integrable function on the cube $[0,1)^{d}$. We define the associated rectangular partial sums of $d$-dimensional WalshFourier series by the formula

$$
S_{n_{1}, n_{2}, \ldots, n_{d}}(f)(x)=\sum_{j_{1}=0}^{n_{1}-1} \sum_{j_{2}=0}^{n_{2}-1} \ldots \sum_{j_{d}=0}^{n_{d}-1} \hat{f}\left(j_{1}, j_{2}, \ldots, j_{d}\right) \prod_{k=1}^{d} w_{j_{k}}\left(x_{k}\right) .
$$

Here $x=\left(x_{1}, x_{2}, \ldots, x_{d}\right) \in[0,1)^{d}$ and

$$
\hat{f}\left(j_{1}, j_{2}, \ldots, j_{d}\right)=\int_{[0,1)^{d}} f(x) \prod_{k=1}^{d} w_{j_{k}}\left(x_{k}\right) \mathrm{d} x
$$

is the $\left(j_{1}, j_{2}, \ldots, j_{d}\right)$ th Walsh-Fourier coefficient of $f$. The relation between the size of $f$ and the behavior of the partial $S_{n, n, \ldots, n}(f)$ has gained a lot of interest in the literature. For this and closely related topic, consult e.g. the works of Goginava [5,6], Goginava and Weisz [7], Nagy [9], Simon [13,14] and Weisz [21-23]. We will study this interplay from a slightly different point of view. Given a parameter $\alpha \in(0, d)$, consider the associated fractional integral operator $I_{\alpha}$ by

$$
I_{\alpha} f=\sum_{k=0}^{\infty} 2^{-k \alpha} S_{k, k, \ldots, k}(f) .
$$

This is the discrete and localized version of the usual fractional integral operator (Riesz potential) in $\mathbb{R}^{d}$ (see Stein [17]). This object was studied by Watari [20] (a convenient reference, which presents a probabilistic approach, is the paper of Chao and Ombe [2]). For more recent works, we refer the interested reader to the works of Lacey et. al. [8] and Cruz-Uribe and Moen [3]. The arguments presented in these papers can be used to prove that the fractional integral operator is bounded as an operator from $L_{p}\left([0,1)^{d}\right)$ to $L_{q}\left([0,1)^{d}\right)$, where $1<p \leq d / \alpha$ and $\frac{1}{q}=\frac{1}{p}-\frac{\alpha}{d}$. Furthermore, in the limit case $p=1$, $q=d /(d-\alpha)$, we have the weak-type estimate

$$
\left\|I_{\alpha} f\right\|_{L^{q, \infty}\left([0,1)^{d}\right)} \leq C_{\alpha, d}\|f\|_{L^{p}\left([0,1)^{d}\right)},
$$

where

$$
\|f\|_{L^{q, \infty}\left([0,1)^{d}\right)}=\sup _{\lambda>0} \lambda\left|\left\{x \in[0,1)^{d}:|f(x)| \geq \lambda\right\}\right|^{1 / q}
$$

is the usual weak-type quasi-norm. This should be compared to the analogous statements concerning the classical Riesz potentials on $\mathbb{R}^{d}$; see e.g. Stein [17]. 
The principal purpose of the present paper is to derive the optimal value of the weak type constant $C_{\alpha, d}$. Here is the precise statement.

Theorem 1.1. For any $0<\alpha<d$ and any $f \in L^{p}\left([0,1)^{d}\right)$ we have

$$
\left\|I_{\alpha} f\right\|_{L^{d /(d-\alpha), \infty}\left([0,1)^{d}\right)} \leq \frac{2^{d}-1}{\left(2^{d-\alpha}-1\right)\left(2^{\alpha}-1\right)}\|f\|_{L^{1}\left([0,1)^{d}\right)} .
$$

The inequality is sharp for any $\alpha$ and $d$.

The proof of the above statement will exploit an enhancement of the socalled Bellman function method. This technique originates from the theory of stochastic optimal control, and its connection with other areas of mathematics was firstly observed by Burkholder in [1], who studied certain sharp inequalities for martingale transforms. Since then, the method has been intensively developed in the subsequent works of Burkholder and his students (a convenient reference on the subject is the monograph [12] by the author). Furthermore, in the late 1990s, Nazarov, Treil and Volberg [10,11] showed that the method can be exploited in a much wider analytic context. Since the seminal papers, the technique has been used in numerous settings: see e.g. $[4,15,16,18,19]$ and references therein.

Roughly speaking, the Bellman function method enables to deduce a given inequality from the existence of a certain special function, which enjoys some majorization and convexity-type properties. It turns out that in the study of (1.1), one needs an appropriate extension of the method. More precisely, the weak-type inequality will be deduced from the existence of a certain family of special functions. As we hope, this novel modification can be used in the investigation of other related estimates which arise naturally in the area.

A few words about the organization of the paper are in order. The special functions are introduced and studied in the next section. Then, in Section 3, we show how to exploit their properties to obtain the inequality (1.1). The final part of the paper contains the construction of certain extremal functions on $[0,1)^{d}$, which show that for each $\alpha$ and $d$, the constant $C_{\alpha, d}$ cannot be improved.

\section{A Special Function and its Properties}

As announced in the previous section, the proof of (1.1) will depend heavily on the existence of certain special functions. Given a nonnegative integer $n$, consider $B_{n}:\left\{(x, y): y \geq 2^{-n \alpha} x \geq 0\right\} \rightarrow[0,1]$ given by the formula

$$
B_{n}(x, y)=\left[\frac{\left(2^{d}-1\right) x 2^{-(n+1) \alpha}}{\left(2^{d}-1\right) x 2^{-(n+1) \alpha}+(1-y)\left(2^{d-\alpha}-1\right)}\right]^{d /(d-\alpha)}
$$

if $y<1$, and $B_{n}(x, y)=1$ otherwise.

The key convexity-type property of the family $\left\{B_{n}\right\}_{n \geq 0}$ is described in the following statement. 
Lemma 2.1. Let $n \geq 1$ be a fixed integer and fix $x$, y satisfying $0 \leq 2^{-n \alpha} x \leq y$. Then for any numbers $h_{1}, h_{2}, \ldots, h_{d} \in\left[-x,\left(2^{d}-1\right) x\right]$ satisfying $\sum_{k=1}^{2^{d}} h_{k}=0$ we have

$$
B_{n-1}(x, y) \geq \frac{1}{2^{d}} \sum_{k=1}^{2^{d}} B_{n}\left(x+h_{k}, y+2^{-n \alpha} h_{k}\right) .
$$

If $y \geq 1$, then the above estimate is obvious, since the left-hand side is equal to 1 , while all the summands appearing on the left are at most 1 . Therefore, we may assume that $y<1$. We consider two cases.

Proof of (2.1) for $y+2^{-n \alpha}\left(2^{d}-1\right) x \leq 1$. Then we have $y+h_{k} \leq 1$ for all $k$. Consider the function

$$
\xi(h):=B_{n}\left(x+h, y+2^{-n \alpha} h\right), \quad h \in\left[-x,\left(2^{d}-1\right) x\right] .
$$

Let us show that the graph of $\xi$ lies below the line segment joining $(-x, \xi(-x))$ and $\left(\left(2^{d}-1\right) x, \xi\left(\left(2^{d}-1\right) x\right)\right)$. Since the point

$$
\left(0, B_{n-1}(x, y)\right)=\left(1-2^{-d}\right) \cdot(-x, \xi(-x))+2^{-d} \cdot\left(\left(2^{d}-1\right) x, \xi\left(\left(2^{d}-1\right) x\right)\right)
$$

lies on the segment, this will immediately yield (2.1).

To prove the above statement, it suffices to prove the following:

(a) The left-sided derivative $\xi^{\prime}\left(\left(2^{d}-1\right) x\right)$ is larger or equal to the slope of the segment.

(b) We have $\xi^{\prime \prime}(h) \geq 0$ for $h$ close to $-x$.

(c) The derivative $\xi^{\prime \prime}$ changes its sign at most once.

We start with the property (a). Since $\xi(-x)=0$, the slope of the segment joining $(-x, \xi(-x))$ and $\left(\left(2^{d}-1\right) x, \xi\left(\left(2^{d}-1\right) x\right)\right)$ equals

$$
\begin{aligned}
& \frac{\xi\left(\left(2^{d}-1\right) x\right)}{2^{d} x} \\
& \quad=\frac{1}{2^{d} x}\left[\frac{\left(2^{d}-1\right) 2^{d-(n+1) \alpha} x}{\left(2^{d}-1\right) 2^{d-(n+1) \alpha} x+\left(1-y-2^{-n \alpha}\left(2^{d}-1\right) x\right)\left(2^{d-\alpha}-1\right)}\right]^{d /(d-\alpha)} .
\end{aligned}
$$

On the other hand, some tedious, but straightforward calculations show that the left-sided derivative of $\xi$ at $\left(2^{d}-1\right) x$ is equal to

$$
\begin{aligned}
& \frac{d}{d-\alpha}\left[\frac{\left(2^{d}-1\right) 2^{d-(n+1) \alpha} x}{\left(2^{d}-1\right) 2^{d-(n+1) \alpha} x+\left(1-y-2^{-n \alpha}\left(2^{d}-1\right) x\right)\left(2^{d-\alpha}-1\right)}\right]^{\alpha /(d-\alpha)} \\
& \quad \times \frac{\left(2^{d}-1\right) 2^{-(n+1) \alpha}\left(2^{d-\alpha}-1\right)\left(1-y+2^{-n \alpha} x\right)}{\left[\left(2^{d}-1\right) 2^{d-(n+1) \alpha} x+\left(1-y-2^{-n \alpha}\left(2^{d}-1\right) x\right)\left(2^{d-\alpha}-1\right)\right]^{2}} .
\end{aligned}
$$

Therefore, the assertion of (a) is equivalent to

$$
1 \leq \frac{d}{d-\alpha} \cdot \frac{\left(2^{d-\alpha}-1\right)\left(1-y+2^{-n \alpha} x\right)}{\left(2^{d}-1\right) 2^{d-(n+1) \alpha} x+\left(1-y-2^{-n \alpha}\left(2^{d}-1\right) x\right)\left(2^{d-\alpha}-1\right)},
$$

or, after some manipulations,

$$
(d-\alpha)\left(2^{\alpha}-1\right) 2^{d-(n+1) \alpha} x \leq \alpha\left(1-y+2^{-n \alpha} x\right)\left(2^{d-\alpha}-1\right) .
$$


However, we have $1-y \geq 2^{-n \alpha}\left(2^{d}-1\right) x$ (this is the assumption under which we work: see the beginning of the proof). Therefore, we will be done if we show that

$$
(d-\alpha)\left(2^{\alpha}-1\right) 2^{d-(n+1) \alpha} x \leq \alpha 2^{d-n \alpha} x \cdot\left(2^{d-\alpha}-1\right),
$$

or, equivalently,

$$
\frac{1-2^{-\alpha}}{\alpha} \leq \frac{2^{d-\alpha}-2^{-\alpha}}{d}
$$

This follows directly from the estimate $d \geq \alpha$.

We turn our attention to (b) and (c). First write

$$
\begin{aligned}
\xi(h) & =\left(\frac{2^{d}-1}{2^{\alpha}-1}\right)^{d /(d-\alpha)}\left[1+\frac{2^{-n \alpha}\left(1-2^{-\alpha}\right) x-M}{M+2^{-n \alpha}\left(1-2^{-\alpha}\right) h}\right]^{d /(d-\alpha)} \\
& =C[1+g(h)]^{d /(d-\alpha)},
\end{aligned}
$$

where $M=\left(2^{d}-1\right) x 2^{-(n+1) \alpha}+(1-y)\left(2^{d-\alpha}-1\right)$. Therefore,

$$
\xi^{\prime \prime}(h)=\frac{C d}{d-\alpha}(1+g(h))^{(2 \alpha-d) /(d-\alpha)}\left[\frac{\alpha}{d-\alpha}\left(g^{\prime}(h)\right)^{2}+(1+g(h)) g^{\prime \prime}(h)\right] .
$$

However, we derive that

$$
g^{\prime}(h)=\frac{2^{-n \alpha}\left(1-2^{-\alpha}\right)\left(M-2^{-n \alpha}\left(1-2^{-\alpha}\right) x\right)}{\left(M+2^{-n \alpha}\left(1-2^{-\alpha}\right) h\right)^{2}}
$$

and

$$
g^{\prime \prime}(h)=-2 \cdot \frac{2^{-2 n \alpha}\left(1-2^{-\alpha}\right)^{2}\left(M-2^{-n \alpha}\left(1-2^{-\alpha}\right) x\right)}{\left(M+2^{-n \alpha}\left(1-2^{-\alpha}\right) h\right)^{3}} .
$$

Consequently, the sign of $\xi^{\prime \prime}(h)$ is that of

$$
\frac{\alpha}{d-\alpha}\left(M-2^{-n \alpha}\left(1-2^{-\alpha}\right) x\right)-2 \cdot 2^{-n \alpha}\left(1-2^{-\alpha}\right)(x+h) .
$$

Now both (b) and (c) follow at once, since $M>2^{-n \alpha}\left(1-2^{-\alpha}\right) x$ and the above expression is a decreasing linear function of $h$.

Proof of (2.1) for $y+2^{-n \alpha}\left(2^{d}-1\right) x>1$. As previously, put

$$
\xi(h):=B_{n}\left(x+h, y+2^{-n \alpha} h\right), \quad h \in\left[-x,\left(2^{d}-1\right) x\right] .
$$

That is,

$$
\xi(h)=\left[\frac{\left(2^{d}-1\right)(x+h) 2^{-(n+1) \alpha}}{\left(2^{d}-1\right)(x+h) 2^{-(n+1) \alpha}+\left(1-y-2^{-n \alpha} h\right)\left(2^{d-\alpha}-1\right)}\right]^{d /(d-\alpha)}
$$

if $h<(1-y) 2^{n \alpha}$, and $\xi(h)=1$ otherwise. The argument is a slight modification of that used above. First, let us show that the graph of $\xi$ lies below the line passing through the points $(-x, \xi(-x))=(-x, 0)$ and $\left((1-y) 2^{n \alpha}, \xi\left((1-y) 2^{n \alpha}\right)\right)=\left((1-y) 2^{n \alpha}, 1\right)$. The slope of the line is positive, so the majorization is obvious on $\left[(1-y) 2^{n \alpha},\left(2^{d}-1\right) x\right]$. Thus, it suffices to focus on the interval $\left[-x,(1-y) 2^{n \alpha}\right]$, for which it is enough to show that 
(a) The left-sided derivative $\xi^{\prime}\left((1-y) 2^{n \alpha}\right)$ is larger or equal to the slope of the line.

(b) We have $\xi^{\prime \prime}(h) \geq 0$ for $h$ close to $-x$.

(c) The derivative $\xi^{\prime \prime}$ changes its sign at most once on $\left(-x,(1-y) 2^{n \alpha}\right)$.

Actually, the conditions (b) and (c) are proved by repeating the calculations from in the previous case, word-by-word (the formula for $\xi(h)$ for $h \in\left[-x,(1-y) 2^{n \alpha}\right]$ is the same). To show (a), we derive that the slope of the line is $\left(x+(1-y) 2^{n \alpha}\right)^{-1}$, while the left-sided derivative is given by

$$
\frac{d}{d-\alpha} \cdot \frac{\left(2^{d}-2^{\alpha}\right)}{\left(2^{d}-1\right)\left(x+(1-y) 2^{n \alpha}\right)} .
$$

Therefore, the assertion of (a) can be rewritten as

$$
\frac{2^{d}-2^{\alpha}}{d-\alpha} \geq \frac{2^{d}-1}{d}
$$

which follows directly from the estimate $0 \leq \alpha \leq d$. This implies the majorization of the graph of $\xi$. To complete the proof, we will show that the point $\left(0, B_{n-1}(x, y)\right)$ lies above the line joining $(-x, \xi(-x))$ and $\left((1-y) 2^{n \alpha}, \xi\left((1-y) 2^{n \alpha}\right)\right.$. This amounts to saying that $B_{n-1}(x, y)$ is not smaller than

$$
\frac{(1-y) 2^{n \alpha}}{x+(1-y) 2^{n \alpha}} \xi(-x)+\frac{x}{x+(1-y) 2^{n \alpha}} \xi\left((1-y) 2^{n \alpha}\right)=\frac{x}{x+(1-y) 2^{n \alpha}} .
$$

Now, the substitution $w:=(1-y) 2^{n \alpha} / x$ turns the desired inequality into

$$
1+w \geq\left(1+w \frac{2^{d-\alpha}-1}{2^{d}-1}\right)^{d /(d-\alpha)} .
$$

However, the right-hand side is a convex function of $w$, and both sides are equal if $w=0$ or $w=2^{d}-1$. It remains to note that $0 \leq(1-y) 2^{n \alpha} / x<2^{d}-1$ (the left estimate is trivial, the right follows from the assumption under which we work: see the beginning of the proof).

We conclude this section by the following simple statement.

Lemma 2.2. (i) If $n$ is a nonnegative integer and $(x, y)$ lies in the domain of $B_{n}$, then $B_{n}(x, y) \geq \chi_{\{y \geq 1\}}$.

(ii) We have

$$
B_{0}(x, x) \leq\left(\frac{2^{d}-1}{2^{d}-2^{\alpha}}\right)^{d /(d-\alpha)} x^{d /(d-\alpha)}
$$

Proof. (i) This follows directly from the definition.

(ii) We have

$$
\left(B_{0}(x, x)\right)^{(d-\alpha) / d}=\frac{\left(2^{d}-1\right) 2^{-\alpha} x}{2^{d-\alpha}-1+x\left(1-2^{-\alpha}\right)} \leq \frac{2^{d}-1}{2^{d}-2^{\alpha}} x,
$$

which is the claim. 


\section{Proof of (1.1)}

For any $n \geq 0$, let $\mathcal{F}_{n}$ denote the $\sigma$-algebra generated by all dyadic subcubes of $[0,1)^{d}$ which are of measure $2^{-n d}$. It is convenient to split the reasoning into several separate parts.

Step 1. Some reductions. By a straightforward approximation argument, it is enough to prove the weak-type estimate for functions which are simple. Here by simplicity we mean that $f$ is measurable with respect to some $\mathcal{F}_{N}$ (that is, the corresponding sequence $\left(S_{n, n, \ldots, n}(f)\right)_{n \geq 0}$ stabilizes after a finite number of steps). Introduce the difference operators $\left(D_{n}\right)_{n \geq 0}$ given by $D_{0}(f)=S_{0,0, \ldots, 0}(f)$ and

$$
D_{n}(f)=S_{n, n, \ldots, n}(f)-S_{n-1, n-1, \ldots, n-1}(f), \quad n=1,2, \ldots .
$$

Note that for any $n \geq 0$ and any atom $A$ of $\mathcal{F}_{n}$ we have the equality

$$
\int_{A} D_{n+1}(f)(x) \mathrm{d} x=0 .
$$

To see this, note that by the very definition, $D_{n+1} f(x)$ is a linear combination of the products $\prod_{k=1}^{d} w_{j_{k}}\left(x_{k}\right)$ such that at least one of $j_{k}$ 's is equal to $n+1$. The corresponding Walsh function $w_{j_{k}}$, integrated over a dyadic interval of length $2^{-n}$, gives 0 and hence (3.1) follows. In the probabilistic language, (3.1) means that $\left(S_{n, n, \ldots, n}(f)\right)_{n \geq 0}$, considered as a sequence of random variables (on the probability space $\left([0,1)^{d}, \mathcal{B}\left([0,1)^{d}\right),|\cdot|\right)$ ), is a martingale with respect to the dyadic filtration $\left(\mathcal{F}_{n}\right)_{n \geq 0}$. As an immediate consequence, we see that it suffices to show (1.1) for nonnegative functions only. Indeed, the passage from $f$ to $|f|$ does not affect the $L^{1}$-norm; on the other hand, for any $n \geq 0$ we have

$$
\left|S_{n, n, \ldots, n}(f)\right|=\left|\mathbb{E}\left(f \mid \mathcal{F}_{n}\right)\right| \leq \mathbb{E}\left(|f| \mid \mathcal{F}_{n}\right)=S_{n, n, \ldots, n}(|f|),
$$

so $\left|I_{\alpha}(f)\right| \leq I_{\alpha}(|f|)$ and hence $\left\|\left.I_{\alpha}(f)\right|_{L^{q, \infty}\left([0,1)^{d}\right)} \leq\right\| I_{\alpha}(|f|) \|_{L^{q, \infty}\left([0,1)^{d}\right)}$. Thus, from now on, we assume that $f \geq 0$.

Step 2. An alternative definition of $I_{\alpha}(f)$. It will be convenient to express the integral fractional operator in terms of the difference sequence. Namely, note that

$$
\begin{aligned}
I_{\alpha}(f) & =\sum_{n=0}^{\infty} 2^{-n \alpha} S_{n, n, \ldots, n}(f) \\
& =\sum_{n=0}^{\infty} \sum_{k=0}^{n} 2^{-n \alpha} D_{k}(f)=\left(1-2^{-\alpha}\right)^{-1} \sum_{k=0}^{\infty} 2^{-k \alpha} D_{k}(f),
\end{aligned}
$$

after the change of the order of summation. In what follows, we will use the notation

$$
f_{n}=S_{n, n, \ldots, n}(f)=\sum_{k=0}^{n} D_{k}(f), \quad g_{n}=\sum_{k=0}^{n} 2^{-k \alpha} D_{k}(f), \quad n \geq 0 .
$$


Note that both $\left(f_{n}\right)_{n \geq 0},\left(g_{n}\right)_{n \geq 0}$ are simple: for sufficiently large $n$ we have $f_{n}=f$ and $g_{n}=\left(1-2^{-\alpha}\right) I_{\alpha}(f)$. We conclude this part by proving that for any $n \geq 0$ we have

$$
g_{n} \geq 2^{-n \alpha} f_{n}
$$

This follows from an easy induction. Indeed, for $n=0$ we have $g_{0}=f_{0}$; now, assuming that the bound holds true for $n$, we derive that

$$
g_{n+1}=g_{n}+d g_{n+1} \geq 2^{-n \alpha} f_{n}+2^{-(n+1) \alpha} d f_{n+1} \geq 2^{-(n+1) \alpha} f_{n+1} .
$$

Step 3. The use of functions $B_{n}, n \geq 0$. We will prove the following statement: for $n \geq 0$,

$$
\int_{[0,1)^{d}} B_{n}\left(f_{n}(s), g_{n}(s)\right) \mathrm{d} s \leq \int_{[0,1)^{d}} B_{n+1}\left(f_{n+1}(s), g_{n+1}(s)\right) \mathrm{d} s .
$$

Note that the above formula makes sense: by $(3.3)$, the pair $\left(f_{n}, g_{n}\right)$ takes values in the domain of $B_{n}$.

To show (3.4), fix an atom $A$ of $\mathcal{F}_{n}$. Both $f_{n}$ and $g_{n}$ are $\mathcal{F}_{n}$-measurable, and hence constant on $A$ : denote the corresponding values by $x$ and $y$, respectively. On the other hand, let $A_{1}, A_{2}, \ldots, A_{2^{d}}$ be the pairwise disjoint atoms of $\mathcal{F}_{n+1}$ contained in $A$. Then $D_{n+1}(f)$ is constant on each $A_{j}$; put $h_{j}=\left.D_{n+1}(f)\right|_{A_{j}}$. We have $x+h_{j}=\left.f_{n}\right|_{A_{j}} \geq 0$, so

$$
h_{j} \geq-x \text { for all } j \text {. }
$$

Furthermore, (3.1) implies that

$$
\sum_{k=1}^{2^{d}} h_{k}=0
$$

which, combined with (3.5), yields

$$
h_{j}=-\sum_{k \neq j} h_{k} \leq\left(2^{d}-1\right) x .
$$

Thus the assumptions of Lemma 2.1 are satisfied and (2.1) gives

$$
B_{n}(x, y) \geq \frac{1}{2^{d}} \sum_{k=1}^{2^{d}} B_{n+1}\left(x+h_{k}, y+2^{-n \alpha} h_{k}\right)
$$

which is equivalent to

$$
\int_{A} B_{n}\left(f_{n}(s), g_{n}(s)\right) \mathrm{d} s \geq \int_{A} B_{n+1}\left(f_{n+1}(s), g_{n+1}(s)\right) \mathrm{d} s .
$$

It remains to sum over all $A$ to obtain (3.4).

Step 4. Proof of (1.1). Recall that by simplicity of $g$, there is $n$ such that $g_{n}=\left(1-2^{-\alpha}\right) I_{\alpha}(f)$. Now, all that is left is to use Lemma 2.2. Using the first 
part, then (3.4) and finally the second part of that lemma, we obtain

$$
\begin{aligned}
\int_{[0,1)^{d}} \chi_{\left\{\left(1-2^{-\alpha}\right) I_{\alpha}(f) \geq 1\right\}} \mathrm{d} s & =\int_{[0,1)^{d}} \chi_{\left\{g_{n}(s) \geq 1\right\}} \mathrm{d} s \\
& \leq \int_{[0,1)^{d}} B_{n}\left(f_{n}(s), g_{n}(s)\right) \mathrm{d} s \\
& \leq \int_{[0,1)^{d}} B_{0}\left(f_{0}(s), g_{0}(s)\right) \mathrm{d} s \\
& \leq\left(\frac{2^{d}-1}{2^{d}-2^{\alpha}}\right)^{d /(d-\alpha)}\|f\|_{L^{1}\left([0,1)^{d}\right)}^{d /(d-\alpha)} .
\end{aligned}
$$

Here in the last passage we have exploited the fact that $f_{0}$ is identically $\|f\|_{L^{1}\left([0,1)^{d}\right)}$ on $[0,1)^{d}$. Now apply the above estimate to the function (1$\left.2^{-\alpha}\right)^{-1} f / \lambda$ and multiply both sides by $\lambda^{d /(d-\alpha)}$. Taking the supremum over $\lambda$ on the left completes the proof of (1.1).

Remark 3.1. An important observation is in order. Namely, it is possible to base the above reasoning on one special function $B$ only, instead of the whole family $\left(B_{n}\right)_{n \geq 0}$. Indeed, as one easily verifies, we have the equality

$$
B_{n}(x, y)=B\left(2^{-n \alpha} x, y\right),
$$

where $B:\{(x, y): y \geq x \geq 0\} \rightarrow[0,1]$ is given by

$$
B(x, y)=\left[\frac{\left(2^{d-\alpha}-2^{-\alpha}\right) x}{\left(2^{d-\alpha}-2^{-\alpha}\right) x+(1-y)\left(2^{d-\alpha}-1\right)}\right]^{d /(d-\alpha)}
$$

if $y<1$, and $B(x, y)=1$ otherwise. Then the main inequality (2.1) takes the following more natural form in terms of $B$ :

$$
B\left(2^{\alpha} x, y\right) \geq \frac{1}{2^{d}} \sum_{k=1}^{2^{d}} B\left(x+h_{k}, y+h_{k}\right),
$$

if $0 \leq x \leq y, 0 \leq x+h_{k} \leq y+h_{k}$ and $\sum_{k=1}^{2^{d}} h_{k}=0$. The reason why we have decided to present the more complicated approach using the whole family $\left(B_{n}\right)_{n>0}$ is that the above argumentation can be used for general problems, in which the special functions do not enjoy any scaling property of the type (3.8). Furthermore, the technicalities arising in both settings are of comparable complexity and the reduction (3.8) does not really simplify the calculations.

\section{Sharpness}

Now we will construct appropriate extremal example. Let $d \geq 1, N \geq 1$ be fixed integers and consider the function $f=\chi_{\left[0,2^{-N}\right) \times\left[0,2^{-N}\right) \times \ldots \times\left[0,2^{-N}\right)}$ on $[0,1)^{d}$. Furthermore, for any $0 \leq n \leq N$, put $A_{n}=\left[0,2^{-n}\right) \times\left[0,2^{-n}\right) \times \ldots \times$ 
$\left[0,2^{-n}\right)$. Then for each such $n, A_{n}$ is the unique atom of $\mathcal{F}_{n}$ containing $A_{N}$, and therefore

$$
S_{n, n, \ldots, n} f(x)=\frac{\left|A_{N}\right|}{\left|A_{n}\right|}=2^{(n-N) d}
$$

provided $x \in A_{n}$, and $S_{n, n, \ldots, n} f(x)=0$ elsewhere; furthermore, we have $S_{n, n, \ldots, n} f=S_{N, N, \ldots, N} f=f$ for $n \geq N$. These facts can be deduced directly from the very definition of $S_{n, n, \ldots, n}$, or, alternatively, follow from the martingale interpretation of the sequence $\left(S_{n, n, \ldots, n} f\right)_{n \geq 0}$, mentioned in the previous section. Using the above formulas for $S_{n, n, \ldots, n} f$, we see that if $x \in A_{N}$, then

$$
\begin{aligned}
I_{\alpha}(f)(x)=\sum_{n=0}^{\infty} 2^{-n \alpha} S_{n, n, \ldots, n} f(x) & =\sum_{n=0}^{N} 2^{-n \alpha+(n-N) d}+\sum_{n=N+1}^{\infty} 2^{-n \alpha} \\
& =2^{-N d} \cdot \frac{2^{(N+1)(d-\alpha)}-1}{2^{d-\alpha}-1}+\frac{2^{-(N+1) \alpha}}{1-2^{-\alpha}} .
\end{aligned}
$$

Consequently,

$$
\begin{aligned}
\frac{\left\|I_{\alpha}(f)\right\|_{L^{d /(d-\alpha), \infty}\left([0,1)^{d}\right)}}{\|f\|_{L^{1}\left([0,1)^{d}\right)}} & \geq \frac{\left(2^{-N d} \cdot \frac{2^{(N+1)(d-\alpha)}-1}{2^{d-\alpha}-1}+\frac{2^{-(N+1) \alpha}}{1-2^{-\alpha}}\right)\left|A_{N}\right|^{(d-\alpha) / d}}{\left|A_{N}\right|} \\
& =\frac{2^{-N(d-\alpha)}\left(2^{(N+1)(d-\alpha)}-1\right)}{2^{d-\alpha}-1}+\frac{2^{-\alpha}}{1-2^{-\alpha}} .
\end{aligned}
$$

However, the integer $N$ was arbitrary; letting $N \rightarrow \infty$, we see that the latter expression converges to

$$
\frac{2^{d-\alpha}}{2^{d-\alpha}-1}+\frac{2^{-\alpha}}{1-2^{-\alpha}}=\frac{2^{d}-1}{\left(2^{d-\alpha}-1\right)\left(2^{\alpha}-1\right)} .
$$

This shows that the constant in (1.1) is indeed the best possible.

\section{Acknowledgements}

The author would like to thank the referee for the careful reading of the paper and helpful suggestions, which in particular led to Remark 3.1 above. The results were obtained when the author was visiting Purdue University, USA. The research was supported in part by the NCN grant DEC2012/05/B/ST1/00412.

Open Access. This article is distributed under the terms of the Creative Commons Attribution License which permits any use, distribution, and reproduction in any medium, provided the original author(s) and the source are credited.

\section{References}

[1] Burkholder, D.L.: Boundary value problems and sharp inequalities for martingale transforms. Ann. Probab. 12, 647-702 (1984)

[2] Chao, J.-A., Ombe, H.: Commutators on dyadic martingales. Proc. Japan Acad. Ser. A 61, 35-38 (1985) 
[3] Cruz-Uribe, D., Moen, K.: A fractional Muckenhoupt-Wheeden theorem and its consequences. Integr. Equ. Oper. Theory 76, 421-446 (2013)

[4] Dragičević, O., Volberg, A.: Linear dimension-free estimates in the embedding theorem for Schrödinger operators. J. Lond. Math. Soc. 85, 191-222 (2012)

[5] Goginava, U.: Marcinkiewicz-Fejer means of $d$-dimensional Walsh-Fourier series. J. Math. Anal. Appl. 307, 206-218 (2005)

[6] Goginava, U.: The weak type inequality for the maximal operator of the Marcinkiewicz-Fejér means of the two-dimensional Walsh-Fourier series. J. Approx. Theory 154, 161-180 (2008)

[7] Goginava, U., Weisz, F.: Maximal operator of the Fejér means of triangular partial sums of two-dimensional Walsh-Fourier series. Georgian Math. J. 19, 101$115(2012)$

[8] Lacey, M.T., Moen, K., Pérez, C., Torres, R.H.: Sharp weighted bounds for fractional integral operators. J. Funct. Anal. 259, 1073-1097 (2010)

[9] Nagy, K.: Some convergence properties of the Walsh-Kaczmarz system with respect to the Marcinkiewicz means. Rend. Mat. 76, 503-516 (2005)

[10] Nazarov, F.L., Treil, S.R.: The hunt for a Bellman function: applications to estimates for singular integral operators and to other classical problems of harmonic analysis. St. Petersburg Math. J. 8, 721-824 (1997)

[11] Nazarov, F.L., Treil, S.R., Volberg, A.: The Bellman functions and two-weight inequalities for Haar multipliers. J. Am. Math. Soc. 12, 909-928 (1999)

[12] Osȩkowski, A.: Sharp martingale and semimartingale inequalities. Monografie Matematyczne, vol. 72. Birkhäuser, Basel (2012)

[13] Simon, P.: Cesáro summability with respect to two-parameter Walsh system. Monatsh. Math. 131, 321-334 (2000)

[14] Simon, P.: Cesáro means of Vilenkin-Fourier series. Publ. Math. Debrecen 59, 203-219 (2001)

[15] Slavin, L., Stokolos, A., Vasyunin, V.: Monge-Ampère equations and Bellman functions: The dyadic maximal operator. C. R. Acad. Sci. Paris Ser. I 346, 585588 (2008)

[16] Slavin, L., Vasyunin, V.: Sharp results in the integral-form John-Nirenberg inequality. Trans. Am. Math. Soc. 363, 4135-4169 (2011)

[17] Stein, E.M.: Singular Integrals and Differentiability Properties of Functions. Princeton University Press, Princeton (1970)

[18] Vasyunin, V.: The exact constant in the inverse Hölder inequality for Muckenhoupt weights (Russian). Algebra i Analiz 15, 73-117 (2003); translation in St. Petersburg Math. J. 15, 49-79 (2004)

[19] Vasyunin, V., Volberg, A.: Monge-Ampére equation and Bellman optimization of Carleson Embedding Theorems. Linear and Complex Analysis. Am. Math. Soc. Transl. (2), vol. 226, pp. 195-238 (2009)

[20] Watari, C.: Multipliers for Walsh Fourier series. Tohoku Math. J. 16, 239251 (1964)

[21] Weisz, F.: Cesaro summability of two-dimensional Walsh-Fourier series. Trans. Am. Math. Soc. 348, 2169-2181 (1996)

[22] Weisz, F.: Cesáro summability of one and two-dimensional Walsh-Fourier series. Anal. Math. 22, 229242 (1996) 
[23] Weisz, F.: Summability of Multi-Dimensional Fourier Series and Hardy Spaces. Math. Appl. Kluwer Academic, Dordrecht 2002

[24] Zygmund, A.: Trigonometric Series, vol. 2. Cambride University Press, London (1968)

Adam Osȩkowski $(\bowtie)$

Department of Mathematics

Informatics and Mechanics

University of Warsaw

Banacha 2

02-097 Warsaw

Poland

e-mail: ados@mimuw.edu.pl

Received: September 29, 2013.

Revised: December 1, 2013. 